(1)

CrossMark

\title{
New Surviving Sepsis Campaign guidelines: back to the art of medicine
}

\author{
Raffaele Scala ${ }^{1}$, Marcus Schultz ${ }^{2,3}$, Lieuwe D.J. Bos (i) $^{2,4}$ and Antonio Artigas ${ }^{5}$ \\ Affiliations: ${ }^{1}$ Pulmonology and Respiratory Intensive Care Unit, S. Donato Hospital, Arezzo, Italy. ${ }^{2}$ Dept of \\ Intensive Care, Academic Medical Center, Amsterdam, the Netherlands. ${ }^{3}$ Mahidol-Oxford Tropical Medicine \\ Research Unit, Mahidol University, Bangkok, Thailand. ${ }^{4}$ Dept of Respiratory Medicine, Academic Medical \\ Center, Amsterdam, the Netherlands. ${ }^{5}$ Corporación Sanitaria Universitaria Parc Tauli, CIBER Enfermedades \\ Respiratorias, Autonomous University of Barcelona, Sabadell, Spain.
}

Correspondence: Raffaele Scala, Pulmonology and Respiratory Intensive Care Unit, S. Donato Hospital, 52100, Arezzo, Italy. E-mail: raffaele_scaladahotmail.com

@ERSpublications

Surviving Sepsis Campaign guidelines represent an advanced step in the implementation of precision medicine in this field of respiratory and intensive care medicine, where evidence-based recommendations are integrated with best-practice suggestions http://ow.ly/hlk230ko1GN

Cite this article as: Scala R, Schultz M, Bos LDJ, et al. New Surviving Sepsis Campaign guidelines: back to the art of medicine. Eur Respir J 2018; 52: 1701818 [https://doi.org/10.1183/13993003.01818-2017].

Despite the gain in terms of hospital survival achieved in the last decades though the application of Surviving Sepsis Campaign (SSC) guidelines, the treatment of sepsis remains challenging for clinicians as more than one in four septic patients is still likely to die in hospital. In this editorial, we would like to remark on the most important novelty introduced by the new SSC guidelines [1] that we believe represent an advanced step in the implementation of precision medicine in this field of respiratory and intensive care medicine. Although is sensible that the SSC evidence-based recommendations remain a "must" in the bundled usual care of the majority of septic patients, it should be borne in mind that the comprehensive panel of the advice provided by these guidelines might not fit all cases in all clinical scenarios [1]. Accordingly, an individualised approach based on best-practice statements is likely to be coherently considered in these specific circumstances. We therefore want to highlight the existing obstacles that limit the capability of these new guidelines of having a strong impact on clinical outcomes of sepsis around the world [2].

Sepsis is a life-threatening organ dysfunction caused by an infection-induced dysregulated host response, which may be complicated by septic shock when circulatory and cellular/metabolic dysfunction occur [3, 4]. Sepsis and septic shock are the major causes of morbidity and mortality in the world [4, 5]. Prevalence of sepsis varies from $6 \%$ to $30 \%$ among intensive care units (ICUs) depending on sepsis definitions used [3-8]. The population-based incidence of sepsis is estimated to be 290-300 cases per 100000 inhabitants per year in large American series, half of which cases require ICU admission and one-fifth mechanical ventilation [5-7]. National Registry data collected in some European countries reported hospitalisation rate for sepsis of 86-367 cases per 100000 inhabitants per year, with one-third of cases receiving ICU care and a hospital mortality rate of $12-43 \%$ [8-10]. Mortality from sepsis/septic shock differs across continents, countries and regions, with reported in-hospital mortality ranging between $12 \%$ and $76 \%$ [5-13].

Sepsis is a challenging issue not only for intensivists but also for physicians working in pulmonology wards, respiratory high-dependency units (RHDUs) and emergencies departments.

Concerning emergency departments, there was an increase from 1.2 to 2.2 million in the annual admissions for sepsis between 2001 and 2009 in the USA [14]. Sepsis/septic shock is a strong prognostic 
factor in RHDUs, accounting for 36\% of the causes of death in severe acute respiratory failure (ARF) [15]. In the clinical setting of a tremendously increasing burden of critically ill respiratory patients, RHDUs provide a specialised quality of care for ARF patients who require an intermediate level of care between ICU and wards, with health resources optimisation in Europe [16]. The peculiarity of these units is their predominant choice of providing noninvasive monitoring and assistance by means of noninvasive ventilation (NIV) and high-flow nasal therapy to care for patients at earlier stages of ARF. As a matter of the fact, the chance of acquiring nosocomial infections (e.g. ventilator-acquired pneumonia) and sepsis is lower in patients admitted to RHDCUs compared to those requiring invasive monitoring and mechanical ventilation in ICUs [17]. The potential benefit of RHDCU management of the very early phases of sepsis complicating ARF might be due to the use of fewer invasive devices $[16,17]$. In the case that a septic patient does not improve or fulfils the criteria of severe septic shock with multiple organ failure requiring a higher level of care, patients can be transfer to a higher level of ICU care. Unfortunately, RHDCUs do not have an even distribution in Europe and show large differences among countries in terms of number, levels of care provided and structural hospital model; a trend towards an increase in their number and expertise has been reported only in some countries [18].

In 2002, this worldwide health phenomenon prompted a the global initiative, the SSC, with the aim of reducing mortality by $25 \%$ in 5 years, and improving awareness, diagnosis and treatment of sepsis [19-24].

Similar to what has been done for other time-dependent acute illness (i.e. polytrauma, acute myocardial infarction and stroke), the SSC has strongly emphasised the importance of speed and appropriateness of therapy in improving outcomes. Since the application of the first SSC guidelines, a substantial reduction in mortality has been reported [12, 21-23, 25-29]. A recent Spanish multicentre study [29] demonstrated that although the incidence of sepsis/septic shock remained unchanged during a 10-year period, implementation of SSC guidelines decreased the severity of illness and overall mortality. Mortality of sepsis has been shown to be correlated with pre-ICU/RHDCU admission site [13, 15]. Patients with sepsis/septic shock identified in emergency departments, general wards and ICUs in Europe were more severely ill than those in the USA with similar mortality if adjusted for severity of illness and organ dysfunction. Septic patients admitted to European ICUs were more frequently transferred from hospital wards, whereas those in USA were more likely to be admitted directly to ICUs [27]. Similarly, survival is greater in patients admitted to RHDCU as a "step down" from the ICU or emergency department than in those admitted as a "step up" from the wards. This discrepancy could be explained by the fact that the latter patients were more severely ill than the former because they developed a progressive worsening of their conditions while being outside a protected environment [15]. This risk is greater for septic patients whose prognosis is strongly dependent on the prompt application of SSC bundles.

Unfortunately, there are still relevant barriers to the large-scale implementation of effective SSC guidelines [26]. Increased awareness of sepsis as a global healthy priority by governments and contextualisation of guidelines to the particular requirements of low-income countries constitute the key points to improve the worldwide fight against sepsis $[2,30]$.

What strategic advantages emerge from the new SCC bundles [1] compared to the previous ones? First of all, the authors have stressed the meaning of recommendations as the translation of a distillation of the current literature into a coherent set of recommendations suitable for the "typical" septic patients included in the randomised controlled trials (RCTs). In other words, the art of medicine remains a precious ingredient in the personalisation of the application of recommendations to each real-life scenario. Challenging situations have to be approached with precision medicine, which includes interpretation of data and individualisation of treatment [31]. The new SSC guidelines provide guidance at the bedside to a clinical decision maker who is busy and pressured to see more patients in less time. Concerning resuscitation, the new guidelines emphasise the importance of dynamic targets with frequent haemodynamic reassessment through bedside clinical-physiological examination. While the strongly recommended target (mean arterial pressure (MAP) $65 \mathrm{mmHg}$ ) gives benefit for typical patients in early resuscitation, higher targets could be appropriate for the so-called atypical patients that fall outside the RCT-based inclusion criteria, showing, for example, either chronic, poorly controlled hypertension, intra-abdominal compartment syndrome or acute renal hypoperfusion, for whom it is reasonable to achieve higher haemodynamic targets [26].

Since not all patients exhibit the same cardiovascular comorbidities, the recommendation given for resuscitation $\left(30 \mathrm{~mL} \cdot \mathrm{kg}^{-1}\right.$ fluid therapy in $\left.3 \mathrm{~h}\right)$ might result in under-resuscitation in some patients or over-resuscitation in others. A more individualised fluid strategy during early sepsis is necessary to achieve levels of blood pressure and cardiac output compatible with immediate survival [1, 31]. The recommendation of administering $30 \mathrm{~mL} \cdot \mathrm{kg}^{-1}$ is an arbitrary amount of fluid for initial resuscitation and $3 \mathrm{~h}$ is a too long period of time to evaluate the haemodynamic response. A more moderate amount of 


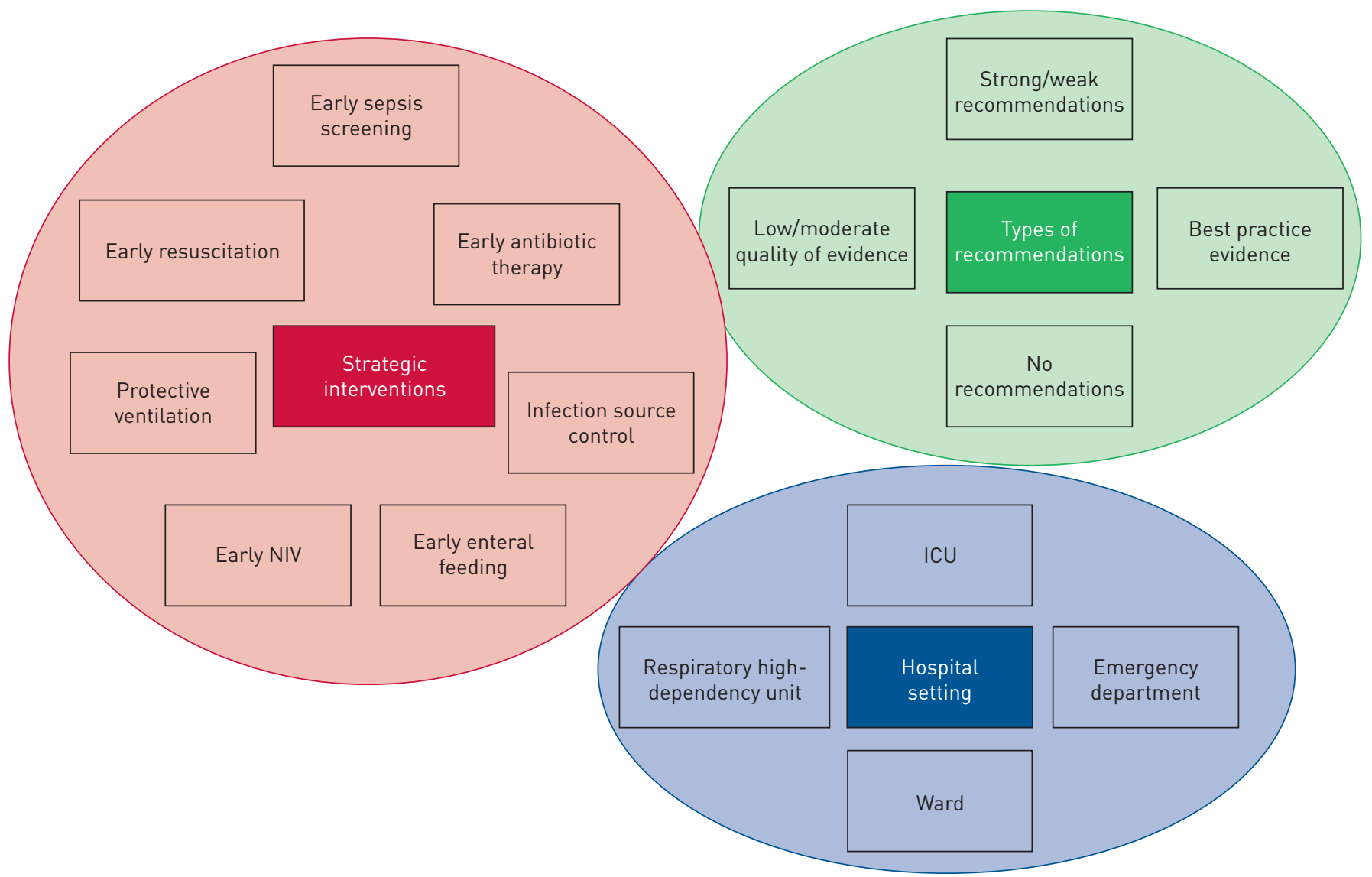

FIGURE 1 Strategic interventions, types of recommendations and setting for sepsis management according to the new Surviving Sepsis Campaign guidelines. NIV: noninvasive ventilation; ICU: intensive care unit.

fluid (e.g. $10 \mathrm{~mL} \cdot \mathrm{kg}^{-1}$ ) is recommended especially in septic patients with acute respiratory distress syndrome or with cardiac or renal failure, evaluating the response in $1 \mathrm{~h}$. The simultaneous use of vasoactive drugs during resuscitation is now recommended to avoid a delay to start vasoactive support to keep the target MAP $[32,33]$.

Moreover, this new guidance takes in consideration sepsis management in different hospital settings, not only in the ICU but also in the emergency department and on the wards. The recommendation of a quick $30 \mathrm{~mL} \cdot \mathrm{kg}^{-1}$ crystalloid infusion within the first $3 \mathrm{~h}$ fits the majority of patients in every environment [26].

Second, these guidelines may be variably read according to different depth of concentric layers (statement, rationale and tables), similar to an onion, depending on the expertise and level of interest of the clinician, as well as the reasons (e.g. educational or research) for their application [31].

Third, the new guidelines have modified the methodology for grading the recommendations. Instead of ungraded recommendations, the 2016 SSC guidelines introduced the best-practice statement [1]. These recommendations (i.e. starting resuscitation immediately) is based on the fact that even though they lack evidence-based literature, which will probably never be available, their effects are reasonably judged to be undoubtedly either beneficial or harmful by clinicians according to their daily practice [34]. An example of individualised care is given by the identification of two ARDS subphenotypes that respond differently to fluid resuscitation [35]. Furthermore, the strength of literature-based recommendations has implications for both clinicians and patients. For what strong recommendations concern, patients would accept that intervention and most clinicians should use it in most "typical" situations. However, on an individual basis, even strong recommendation cannot or should not be followed because patient's wishes or clinical features falling outside RCT scenarios make the recommended intervention less/not applicable.

Fourth, compared to the previous ones, these new guidelines emphasise much more the importance of early sepsis recognition and appropriate treatment among the five areas of management (haemodynamics, infection, adjunct therapies, metabolic and ventilation) (figure 1). Prognosis of sepsis/septic shock is 
TABLE 1 Main changes in the 2016 Surviving Sepsis Campaign recommendations from the 2012 guidelines

Initial resuscitation

Screening for sepsis and performance improvement Antimicrobial therapy

\section{Mechanical ventilation}

Stress ulcer prophylaxis

Nutrition
Initial resuscitation with $\geqslant 30 \mathrm{~mL} \cdot \mathrm{kg}^{-1}$ intravenous crystalloid fluid given within the first $3 \mathrm{~h}$

with following infusion of additional fluids guided by frequent reassessment of haemodynamic status

Implementation of hospital systems to improve programme for sepsis, including sepsis screening for acutely ill, high-risk patients

Optimisation of antimicrobial doses based on pharmacokinetic/ pharmacodynamic principles and specific drug properties

Avoidance of combination therapy for the routine treatment of neutropenic sepsis/bacteraemia

Use of procalcitonin levels to support shortening the duration of antimicrobial therapy in sepsis patients

Application of an upper limit goal for plateau pressures of $30 \mathrm{~cm} \mathrm{H}_{2} \mathrm{O}$ over higher plateau pressures in adult patients with sepsis-induced severe ARDS

Ventilation strategy of prone over supine position in adult patients with sepsis-induced ARDS and a $\mathrm{PaO}_{2} / \mathrm{FlO}_{2}$ ratio $<150$

Use of noninvasive ventilation for patients with sepsis-induced ARDS

Alternative choice of either proton pump inhibitors or histamine-2 receptor antagonists for ulcer prophylaxis

Alternative choice of either early trophic/hypocaloric (with tolerance-guided increase in feeding supply) or early full enteral feeding

Use of prokinetic agents in patients with feeding intolerance

Placement of post-pyloric feeding tubes in patients with feeding intolerance or at high risk of aspiration
Strong recommendation, low

quality of evidence BPS

BPS

BPS

Strong recommendation, moderate quality of evidence

Weak recommendation, low quality of evidence

Strong recommendation, moderate quality of evidence

Strong recommendation, moderate quality of evidence

No recommendation

Weak recommendation, low quality of evidence

Weak recommendation, moderate quality of evidence

Weak recommendation, low quality of evidence

Weak recommendation, low quality of evidence

ARDS: acute respiratory distress syndrome; $\mathrm{PaO}_{2}$ : arterial oxygen tension; $\mathrm{FlO}_{2}$ : inspiratory oxygen fraction; BPS: best-practice statement.

terribly dependent on the immediateness of treatment, such as administration of empiric broad-spectrum antibiotic therapy within $1 \mathrm{~h} \mathrm{[36]} \mathrm{and} \mathrm{infection} \mathrm{source} \mathrm{control} \mathrm{[37].}$

Among the main changes in the new guidelines (table 1), it is worth mentioning those concerning ventilator strategies in sepsis-induced ARDS. Pneumonia is the most frequent cause of sepsis and is related to a worse outcome [3-8, 14, 15, 37]. In addition to slight changes of recommendations for invasive mechanical ventilation (IMV) (i.e. protective ventilation plus high positive end-expiratory pressure levels, and prone ventilation in severe hypoxaemia), which are similar to those applied to nonseptic ARDS patients, dynamic reassessment of fluid administration according to fluid responsiveness, gas-exchange evolution and extravascular lung water to prevent excessive fluid overload has been highlighted to avoid deterioration of the initial sepsis-induced lung injury $[1,38]$. Concerning the role of NIV, the new guidelines keep a neutral position (no recommendation), leaving potential room for this technique in specific categories of patients handled in expert settings [1]. The strong rationale in favour of NIV (i.e. early application, prevention of IMV-induced complications and management outside an overcrowded ICU) should be balanced against its risks, which are greater in de novo acute hypoxaemic patients (i.e. interface intolerance and skin breakdown after prolonged ventilation, inability to cope with abundant secretions, and difficulty applying ARDS-protective ventilatory strategies) [38]. Before stronger evidence is available, the use of NIV in hypoxaemic septic patients should be guided by bedside application of precision medicine with dynamic evaluation of pro/con arguments, intensity and level of team expertise, and employment of integrated strategies (i.e. analgosedation, bronchoscopy, interface rotation, etc.) [39].

Fifth, it should be emphasised that many of the SSC recommendations are not available, affordable or safe in resource-poor settings. This is due to the differences in sepsis management in low- compared to high-income countries, such as setting of treatment (non-ICU environment or poorly equipped ICUs), aetiology (i.e. higher prevalence of tropical diseases with direct damaging effects of pathogens, like malaria), and expertise and training of the staff [30].

The guideline authors acknowledged the bias related to the recently published new definitions for sepsis and septic shock [3] while these guidelines have been developed. In the studies included in the guidelines, the patient populations were primarily characterised by the previous definition of sepsis. The new sepsis definition shifts emphasis from the systemic inflammatory response syndrome to organ dysfunction, 
quantified using the a "quick" version of the Sequential Organ Failure Assessment score [4]. Even if this new proposed score is likely to rapidly identify potentially high-risk infected patients such as those with community-acquired pneumonia [40], its utility in identifying subjects with serious infections before frank sepsis ensues is still under debate [41].

In conclusion, these new guidelines turn out to be a "brick in the wall" of the health campaign against the sepsis in the direction of precision medicine. What we should learn is that nothing in the guidance is absolutely true for every patient in every situation. Even if a strong recommendation has to be included in the usual care of the majority of patients, this advice may be not always the best in all individuals. Let us integrate the art of evidenced-based science with that of practically based medicine.

Conflict of interest: L.D.J. Bos reports receiving grants from the Dutch Lung Foundation and European Respiratory Society, outside the submitted work.

\section{References}

1 Rhodes A, Evans LE, Alhazzani W, et al. Surviving Sepsis Campaign: international guidelines for management of sepsis and septic shock: 2016. Intensive Care Med 2017; 43: 304-377.

2 Reinhart K, Daniels R, Kissoon N, et al. Recognizing sepsis as a global health priority - a WHO resolution. N Engl J Med 2017; 377: 414-417.

3 Seymour CW, Liu VX, Iwashyna TJ, et al. Assessment of clinical criteria for sepsis: for the Third International Consensus Definitions for Sepsis and Septic Shock (Sepsis-3). JAMA 2016; 315: 762-774.

4 Singer M, Deutschman CS, Seymour CW, et al. The Third International Consensus Definitions for Sepsis and Septic Shock (Sepsis-3). JAMA 2016; 315: 801-810.

5 Martin GS. Sepsis, severe sepsis and septic shock: changes in incidence, pathogens and outcomes. Expert Rev Anti Infect Ther 2012; 10: 701-706.

6 Angus DC, Linde-Zwirble WT, Lidicker J, et al. Epidemiology of severe sepsis in the United States: analysis of incidence, outcome, and associated costs of care. Crit Care Med 2001; 29: 1303-1310.

7 Machado FR, Cavalcanti AB, Bozza FA, et al. The epidemiology of sepsis in Brazilian intensive care units (the Sepsis PREvalence Assessment Database, SPREAD): an observational study. Lancet Infect Dis 2017; 17: 1180-1189.

8 Alberti C, Brun-Buisson C, Burchardi H, et al. Epidemiology of sepsis and infection in ICU patients from an international multicentre cohort study. Intensive Care Med 2002; 28: 108-121.

9 Esteban A, Frutos-Vivar F, Ferguson ND, et al. Sepsis incidence and outcome: contrasting the intensive care unit with the hospital ward. Crit Care Med 2007; 35: 1284-1289.

10 Bouza C, López-Cuadrado T, Saz-Parkinson Z, et al. Epidemiology and recent trends of severe sepsis in Spain: a nationwide population-based analysis (2006-2011). BMC Infect Dis 2015; 14: 717.

11 Knoop ST, Skrede S, Langeland N, et al. Epidemiology and impact on all-cause mortality of sepsis in Norwegian hospitals: A national retrospective study. PLoS One 2017; 12: e0187990.

12 Herrán-Monge R, Muriel-Bombín A, García-García MM, et al. Epidemiology and changes in mortality of sepsis after the implementation of Surviving Sepsis Campaign Guidelines. J Intensive Care Med 2017; in press [https:// doi.org/10.1177/0885066617711882].

13 Motzkus CA, Chrysanthopoulou SA, Luckmann R, et al. ICU Admission Source as a Predictor of Mortality for Patients With Sepsis. J Intensive Care Med 2017; in press [https://doi.org/10.1177/0885066617701904].

14 Herring AA, Ginde AA, Fahimi J, et al. Increasing critical care admissions from U.S. emergency departments, 2001-2009. Crit Care Med 2013; 41: 1197-1204.

15 Valentini I, Pacilli AM, Carbonara P, et al. Influence of the admission pattern on the outcome of patients admitted to a respiratory intensive care unit: does a step-down admission differ from a step-up one? Respir Care 2013; 58: 2053-2060.

16 Scala R. Respiratory high-dependency care units for the burden of acute respiratory failure. Eur J Intern Med 2012; 23: 302-308.

17 Girou E, Brun-Buisson C, Taillé S, et al. Secular trends in nosocomial infections and mortality associated with noninvasive ventilation in patients with exacerbation of COPD and pulmonary edema. JAMA 2003; 290: 2985-2991.

18 Scala R, Corrado A, Confalonieri M, et al. Increased number and expertise of Italian respiratory high-dependency care units: the second national survey. Respir Care 2011; 56: 1100-1107.

19 Dellinger RP, Carlet JM, Masur H, et al. Surviving Sepsis Campaign guidelines for management of severe sepsis and septic shock. Intensive Care Med 2004; 30: 536-555.

20 Dellinger RP, Levy MM, Carlet JM, et al. Surviving Sepsis Campaign: international guidelines for management of severe sepsis and septic shock: 2008. Crit Care Med 2008; 36: 296-327.

21 Dellinger RP, Levy MM, Rhodes A, et al. Surviving Sepsis Campaign: international guidelines for management of severe sepsis and septic shock: 2012. Crit Care Med 2013; 41: 580-637.

22 Ferrer R, Artigas A, Levy MM, et al. Improvement in process of care and outcome after a multicenter severe sepsis educational program in Spain. JAMA 2008; 299: 2294-2303.

23 Levy MM, Dellinger RP, Townsend SR, et al. The Surviving Sepsis Campaign: results of an international guideline-based performance improvement program targeting severe sepsis. Crit Care Med 2010; 38: 367-374.

24 Castellanos-Ortega A, Suberviola B, Garcia-Astudillo LA, et al. Impact of the Surviving Sepsis Campaign protocols on hospital length of stay and mortality in septic shock patients: results of a three-year follow-up quasi-experimental study. Crit Care Med 2010; 38: 1036-1043.

25 Yealy DM, Kellum JA, Huang DT, et al. A randomized trial of protocol-based care for early septic shock. N Engl J Med 2014; 370: 1683-1693.

26 ARISE Investigators, ANZICS Clinical Trials Group, Peake SL, et al. Goal-directed resuscitation for patients with early septic shock. N Engl J Med 2014; 371: 1496-1506. 
27 Levy MM, Artigas A, Phillips GS, et al. Outcomes of the Surviving Sepsis Campaign in intensive care units in the USA and Europe: a prospective cohort study. Lancet Infect Dis 2012; 12: 919-924.

28 Blanco J, Muriel-Bombín A, Sagredo V, et al. Incidence, organ dysfunction and mortality in severe sepsis: a Spanish multicentre study. Crit Care 2008; 12: R158.

29 van Zanten ARH, Brinkman S, Arbous MS, et al. Guideline bundles adherence and mortality in severe sepsis and septic shock. Crit Care Med 2014; 42: 1890-1898.

30 Schultz MJ, Dunser MW, Dondorp AM, et al. Current challenges in the management of sepsis in ICUs in resource-poor settings and suggestions for the future. Intensive Care Med 2017; 43: 612-624.

31 Dellinger RP, Schorr CA, Levy MM. A users' guide to the 2016 Surviving Sepsis Guidelines. Intensive Care Med 2017; 43: 299-303.

32 Futier E, Lefrant JY, Guinot PG, et al. Effect of individualized vs standard blood pressure management strategies on postoperative organ dysfunction among high-risk patients undergoing major surgery: a randomized clinical trial. JAMA 2017; 318: 1346-1357.

33 Marik PE, Linde-Zwirble WT, Bittner EA, et al. Fluid administration in severe sepsis and septic shock, patterns and outcomes: an analysis of a large national database. Intensive Care Med 2017; 43: 625-632.

34 Guyatt GH, Schunemann HJ, Djulbegovic B, et al. Guideline panels should not GRADE good practice statements. J Clin Epidemiol 2015; 68: 597-600.

35 Famous KR, Delucchi K, Ware LB, et al. Acute respiratory distress syndrome subphenotypes respond differently to randomized fluid management strategy. Am J Respir Crit Care Med 2017; 195: 331-338.

36 Rochwerg B, Alhazzani W, Sindi A, et al. Fluid resuscitation in sepsis: a systematic review and network meta-analysis. Ann Intern Med 2014; 161: 347-355.

37 Martínez ML, Ferrer R, Torrents E, et al. Impact of source control in patients with severe sepsis and septic shock. Crit Care Med 2017; 45: 11-19.

38 Demoule A, Hill N, Navalesi P. Can we prevent intubation in patients with ARDS? Intensive Care Med 2016; 42: $768-771$.

39 Scala R. Challenges on non-invasive ventilation to treat acute respiratory failure in the elderly. BMC Pulm Med 2016; 16: 150.

40 Ranzani OT, Prina E, Menéndez R, et al. New sepsis definition (Sepsis-3) and community-acquired pneumonia mortality. A validation and clinical decision-making study. Am J Respir Crit Care Med 2017; 196: 1287-1297.

41 Rhee C, Klompas M. New sepsis and septic shock definitions: clinical implications and controversies. Infect Dis Clin North Am 2017; 31: 397-413. 